\title{
La teoría del valor-trabajo a través de las principales escuelas económicas: un enfoque documental
}

\section{The value-work theory through the main economic schools: a documentary approach}

Wilson Castro Yatacue, Paola Espitia Montaña, Sofía Gallego Ruiz, Jorge Galvis Sánchez, Diana Gutiérrez Valencia, Laura Jiménez Cadena y Cristian Ortega Medina*

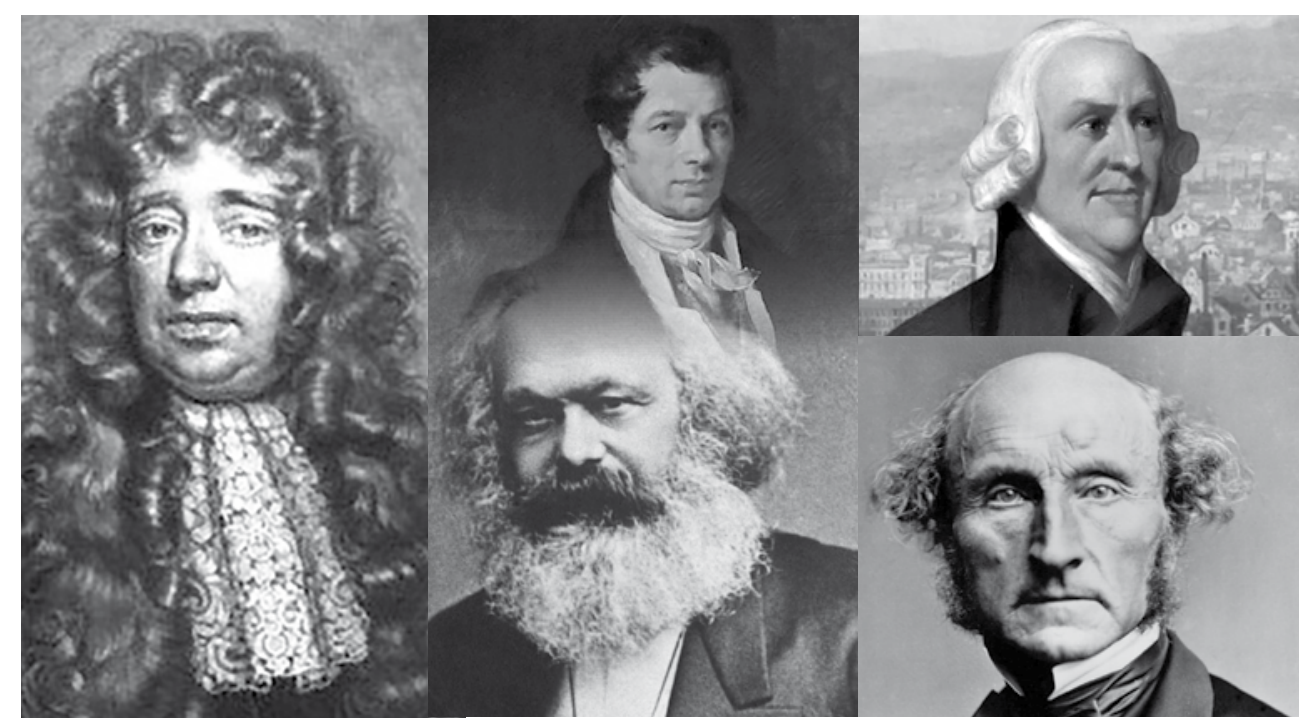

\section{Resumen}

Este trabajo hace una exposición, basada en una recopilación documental, sobre la visión de la teoría del valor-trabajo de los autores más representativos de las diferentes escuelas de pensamiento económico desde el siglo XVII con William Petty,

Citar este artículo como: Castro, W., Espitia, P., Gallego, S., Galvis, J., Gutiérrez Valencia, D., Jiménez, L. y Ortega, C. (2020). La teoría del valor-trabajo a través de las principales escuelas económicas: un enfoque documental. Revista Papeles, 11(22), 89-105.

Fecha de recibido: septiembre 20 de 2019 • Fecha de aceptación: noviembre 30 de 2019

* Estudiantes del curso de Metodología de la Investigación I de la Universidad Nacional de Colombia, sede Bogotá, durante el periodo 2019-II dirigidos por el profesor Enrique Ferrer-Corredor. Correos electrónicos: Wilson Castro (wacastroy@unal.edu.co), Paola Espitia (lespitiam@unal.edu.co), Sofía Gallego (kgallego@unal.edu.co), Jorge Galvis (joregalvissan@unal.edu.co), Diana Gutiérrez (digutierrezv@unal.edu.co), Laura Jiménez (laujimenezca@unal.edu.co), Cristian Ortega (ccortegame@ unal.edu.co). 
pasando por Adam Smith, David Ricardo, Karl Marx, Alfred Marshall, John Keynes, entre otros; esto con la finalidad de determinar la transformación que ha tenido este concepto a través de estos y su evolución histórica. Finalmente, este trabajo concluye que la generalidad de los autores no reconoce una importancia estratégica o mayoritaria al trabajo como único elemento del valor; en cambio, se observa una ponderación entre varios elementos y categorías conceptuales. Adicionalmente, se establece a Marx como punto cúspide de la teoría, y, además, como punto de inflexión en donde la teoría del valor abandona paulatinamente la importancia estratégica del trabajo.

Palabras clave: teoría del valor-trabajo, escuelas económicas, desarrollo conceptual, trabajo.

\section{Abstract}

This work makes an exhibition, based on a documentary compilation, about the vision of the labor theory of value of the most representative authors of the different schools of economic thought, from the 17th century with William Petty, through Adam Smith, David Ricardo, Karl Marx, Alfred Marshall, John Keynes, among others, in order to determine the transformation that this concept has had through these. Finally, this paper concludes that the generality of the authors do not recognize a strategic o majority importance to the work as the only element of value; instead, a weighting between several elements and conceptual categories is observed. Additionally, Marx is established as the apex point of the theory and, in addition, as the inflection point, where the labour theory of value gradually leaves the strategic importance of the labour.

Key words: labour theory of value, schools of economic thought, conceptual development.

\section{Introducción}

La teoría del valor, en la actualidad académica, es una temática comúnmente relegada a los cursos introductorios de económica que, en el mejor de los casos, tratan sobre la teoría del valor contemporánea. De esta manera, se ha excluido su investigación en los campos académicos. Esta investigación retoma la teoría del valor-trabajo desde un análisis histórico con la finalidad de poder quebrantar la caja negra en la cual se ha encerrado a este concepto, demostrar el amplio desarrollo teórico de la misma y extender el discurso de la teoría valor-trabajo a la actualidad.

Ante la amplitud conceptual que presenta este tema, el presente trabajo se centra en identificar y describir las concepciones de la teoría valor-trabajo de los principales autores y escuelas económicas; adicionalmente, se propone contrastar dichas concepciones y determinar el desarrollo conceptual que ha tenido la teoría del valor-trabajo, junto con los períodos de estabilidad, auge y decadencia de esta teoría.

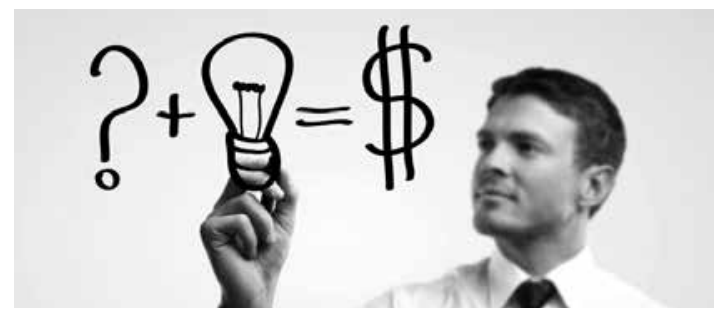




\section{Teoría del valor: una primera aproximación}

Generalmente, la teoría del valor ha oscilado entre dos perspectivas conceptuales, la cuales, según Moreno y Rendón (2005), se han catalogado como teorías del valor objetivas y subjetivas (p. 49). La primera de ellas postula que el valor está determinado por los costos en los que se incurren para la elaboración de las mercancías, mientras que la segunda recae en dimensiones de valoración, momento de consumo y en la marginalidad de utilidad de uso.

El concepto de la teoría del valor-trabajo, perteneciente a la perspectiva objetiva, fue un tema con gran evolución histórica e interés por parte de diferentes académicos y escuelas de pensamiento económico. Es por esto por lo que, en el presente trabajo, solo se observa su evolución dentro de las tres últimas centurias. Para iniciar, aunque, comúnmente, se tenga como introductor de esta teoría a Adam Smith, esta ya se había empezado a constituir en los siglos precedentes. Desde Aristóteles (384-322 a. C.), quien argumentaba una dualidad entre los conceptos de valor de uso y valor de cambio (Jaffe y Lusht, 2003, p. 7), hasta después en el siglo XIII con Albertus Magnus (1205-1280), quien parece haber sido el primero en entrar en la investigación de la teoría del valor-trabajo al sostener que dos productos tenían el mismo valor $\mathrm{y}$, por lo tanto, su intercambio era justo si su producción representaba cantidades iguales de mano de obra y gastos; Santo Tomás de Aquino heredó esta visión (Kaulla, 1940, pp. 37-38). Posteriormente, en William Petty (1623-1687) la fuente del valor recae en la tierra y trabajo; así mismo, establece la distinción entre el valor natural — cantidad de mano de obra necesaria para su producción- y el valor de mercado - determinado por la oferta y la demanda- (Jaffe y Lusht, 2003, p. 15). Esta postura fue compartida por Richard Cantillon (1680-1734) y James Stuart (1806-1873).

Sin embargo, el empleo del trabajo o de los costos de producción como generadores de valor no han sido un consenso entre los académicos a lo largo del tiempo. Por ejemplo, en los antiguos romanos, la teoría del valor se basaba en el grado de estima que tenía un bien (Jaffe y Lusht, 2003, p. 8). Particularmente, Cicerón (106 a. C-43 a. C.) establecía que "the only limit to the valuation of such things is the desire which anyone has for them, for it is difficult to set bounds to the price unless you first

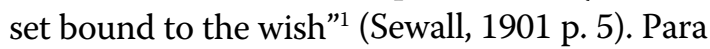
John Hales, la fuente del valor recae en la noción de escasez, comercio y variedad de productos (Jaffe y Lusht, 2003, p. 13). Posteriormente, para Nicholas Barbon el valor se determina por su utilidad de uso de acuerdo las necesidades humanas (Jaffe y Lusht, 2003, pp. 16-17). En último lugar, para John Law el fundamento se encuentra en la proporción de objetos con respecto a su demanda (Jaffe y Lusht, 2003, p. 17-18). De este modo, se evidencia que no ha existido una perspectiva hegemónica a lo largo de la historia, sino que la teoría del valor ha sido un escenario de constante debate.

\section{William Petty (1623-1687)}

Petty, médico y economista inglés, es considerado uno de los precursores de la economía política clásica y uno de los primeros académicos que introdujo el concepto de la teoría del valor- trabajo. Su teoría del valor descansa, como primera instancia, en la influencia de Thomas Hobbes, sobre todo en el Leviatán, en el cual postula que "all commodities come from land and labour"2 (Dooley, 2005, p. 27). Así, en su escrito titulado Treatise of Taxes and Contributions (1963) acepta este postulado al

1 "el único límite para la valoración de tales cosas es el deseo que alguien tiene por ellas, porque es difícil establecer límites al precio a menos que primero establezca límites al deseo" (traducción hecha por los autores).

2 "Todos los productos provienen de la tierra y el trabajo" (traducción hecha por los autores). 


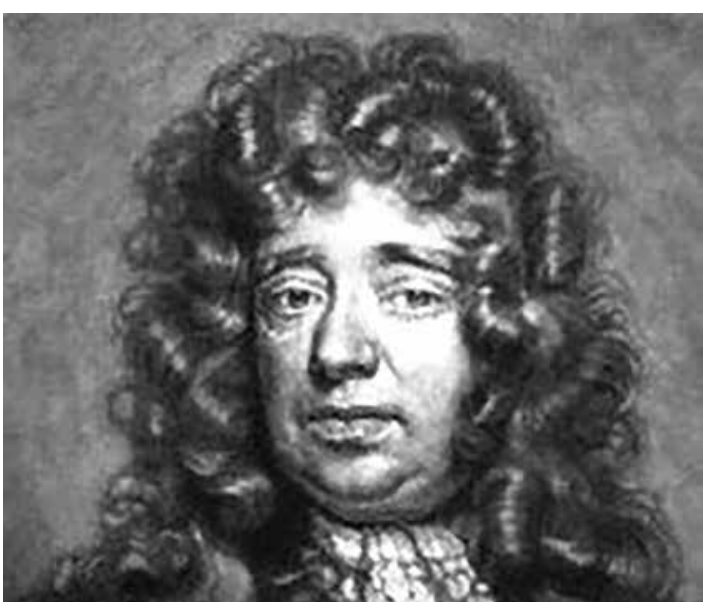

William Petty (1623-1687)

plantear que el "Labour is the Father and active principle of Wealth, as Lands are the Mother" Adicionalmente, en este mismo escrito amplía su teoría respecto a las dos fuentes de valor, apartándose del oro y la plata considerados como portadores de riqueza.

All things ought to be valued by two natural Denominations, which is Land and Labour; that is, we ought to say, a Ship or garment is worth such a measure of Land, with such another measure of Labour; for as-much as both Ships and Garments were the creatures of Lands and means Labours there upon; This being true, we should be glad to find out a natural Par between Land and Labour, so as we might express the value by either of them alone as well or better then by both, and as we reduce pence into pounds ${ }^{4}$ (Petty, 1963, p. 68).

3 "El trabajo es el Padre y principio activo de la Riqueza, como las Tierras son la Madre" (traducción hecha por los autores).

4 "Todas las cosas deben ser valoradas por dos denominaciones naturales, cuales son tierra y trabajo; esto es, debemos decir que un barco o una prenda de vestir tienen el valor de una medida de Tierra, con otra medida de Trabajo; en tanto ambos, los barcos y las prendas, fueron la creación de las Tierras y de los trabajos de los hombres; siendo esto cierto, será
De esta forma, Petty evidencia cómo el valor que aporta el trabajo está representado a través de la producción; de igual forma, la manera en que este último y la tierra producen valor, ya sea de manera independiente $y$, como lo plantea Dooley (2005, p. 27), "it breaks with the Aristotelian tradition (...) that utility, usefulness or demand is the origin of value"

A la vez, distingue dos tipos de valores; el primero, ya mencionado anteriormente, denominado valor interno y el valor de mercado o precio político. Su diferencia recae en que en este último se le incorpora una serie de gastos externos diferenciados del valor natural del producto (tales como factores de demanda o intermediarismo [Díaz, 2014, p. 11]), variando en relación con las fuerzas del mercado. Finalmente, Petty no va más allá de evidenciar los cambios en la magnitud del valor por medio, únicamente, de las condiciones de productividad, pero establece unas bases para su análisis posterior.

\section{Adam Smith (1723-1790): economía clásica}

Adam Smith fue un filósofo y economista inglés, quien nació en 1723 en pleno inicio de la Revolución Industrial. Las bases de su teoría se dan principalmente con la crítica que realizó a los pensadores mercantilistas; así, Dobb nombra que el foco principal fue que, para Smith, ellos persistían en el error de identificar la riqueza con el atesoramiento de dinero, lo cual afectaba el libre desarrollo del mercado

grato encontrar una paridad natural entre tierra y trabajo, así que podríamos expresar el valor solamente por uno de ellos mejor que por ambos, y como nosotros reducimos peniques a libras" (traducción hecha por los autores).

5 "Rompe con la tradición aristotélica (...) que la utilidad, el beneficio o la demanda es el origen del valor" (traducción hecha por los autores). 


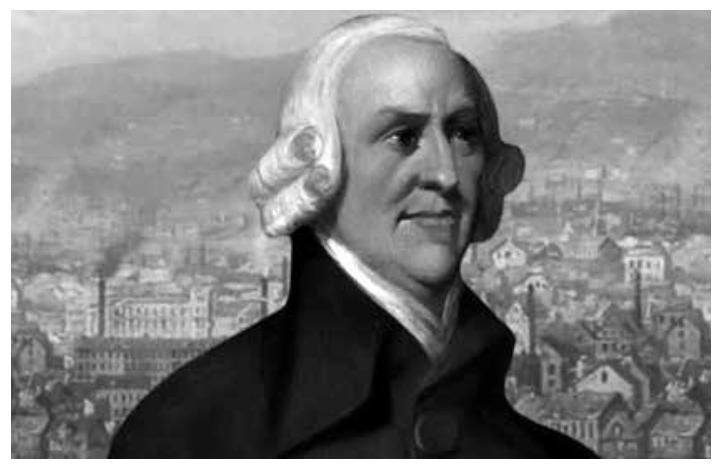

Adam Smith

debido a las inestabilidades que esto generaba (Dobb, 1975p. 71).

Parte del trabajo de Smith fue hacer la diferenciación entre el valor uso y el valor de cambio de las mercancías. Según él, el primero expresa la utilidad y el segundo el poder de compra de estas (Smith, 1994, p. 62). De este último concepto nació su idea que establece el trabajo como el único patrón de medida posible, y la principal razón que da para ello es que iguales cantidades de trabajo en distintas épocas de la historia han significado el mismo esfuerzo para quien las haya realizado, por lo que se torna invariante y constituye un punto de comparación (Dobb, 1975, p. 63).

Para otros autores como Guillén, Adam Smith a través de su teoría, trata de explicar el valor en un entorno un poco adelantado a su época - como se ha dicho antes él vivió en un contexto de capitalismo naciente- de esta forma, mientras que sus predecesores, los fisiócratas, veían que el excedente se daba en la naturaleza, Smith, trató de enlazar el problema en el marco de las relaciones de producción (Guillén, 1976, p. 20). De esa forma es como plantea la "teoría de la suma", la cual es, en síntesis, la agregación de los tres componentes primarios del precio (Dobb, 1975, pp. 59-60); a saber: los salarios, el beneficio y la renta. Esta es una de las razones por las cuales se ha dicho que Adam Smith dio las bases para tener en cuenta la producción y desarrollar la teoría los costos de la misma dentro del análisis económico.

\section{David Ricardo (1772-1823): economía clásica}

David Ricardo, al igual que Adam Smith, fue un economista inglés, pero quien nació en 1772. El principal aporte que da David Ricardo a la teoría del valor-trabajo fue el principio de escasez, ya que reconoce que el trabajo no siempre es el que les da el valor a las mercancías; existen otros factores que hacen que ese valor cambie. Por lo cual, el valor depende de la relación que existe entre trabajo y escasez. Según Eric Roll (1973), la teoría del valor de Ricardo se puede explicar así:

Ricardo, guiado por la teoría de Smith, explica que el valor se puede encontrar en la producción como en el proceso anterior de la mercancía, es decir, en el trabajo presente y pasado. Acepta el valor de uso de Smith y agrega que valor de cambio depende de la escasez o el trabajo, donde todos los objetos tienen diverso valor dependiente de estas dos variantes (Roll, 1973, pp. 177-178).

David Ricardo basó toda su teoría del valortrabajo en el precio de los granos, que en esa época fue muy discutido por las políticas de aranceles, y por lo que posteriormente él plantearía como la calidad de las tierras o "teoría de la renta diferencial”. Estos dos factores, según Ricardo, afectan las rentas y los beneficios de los empresarios, de lo que a su vez dependían los salarios de los trabajadores. Esta teoría fue

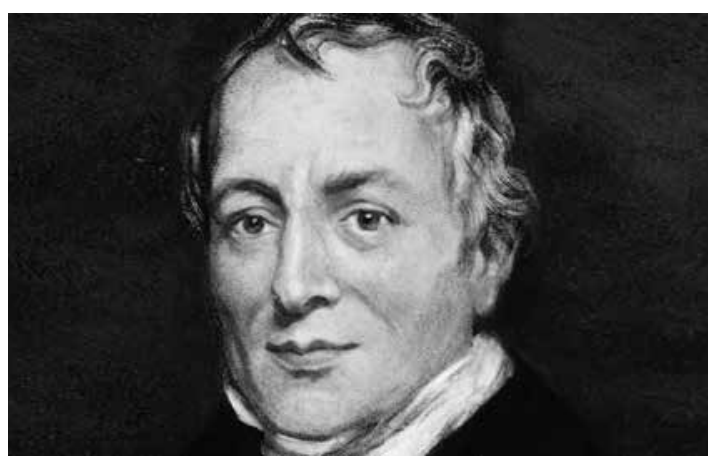

David Ricardo 
otra forma de explicar la teoría de la suma de Adam Smith, pero desde un contexto más agrícola y político. A pesar de esto, Ricardo planteó dos críticas principales a la teoría del valor-trabajo de Adam Smith, a saber: primero, tuvo una confusión entre la cantidad de trabajo y trabajo necesario; en segundo lugar, tuvo un problema en el desarrollo del argumento que planteaba que la teoría del valor depende de la cantidad relativa de trabajo que es necesaria para la producción y no de la mayor o menor compensación que se paga por ese trabajo (Dobb, 1975, p. 90). Según Dobb, otra crítica a la teoría del valor-trabajo de Adam Smith y su contraste con la teoría de David Ricardo es la siguiente:

Pero hemos visto que, hablando con propiedad, carecía de una teoría de la distribución y lo que contiene una teoría del precio o del valor (en la forma de la suma de los tres componentes) es lógicamente incompleto, ya que la distinción entre precio natural y precio de mercado y su principio de las ventajas netas iguales, sus genuinas contribuciones en esta esfera se sostienen por sí mismas. Por el contrario, en Ricardo: encontramos algo muy diferente: una teoría integrada del valor, del beneficio y de las rentas; sus aspectos o elementos tienen la nitidez y la precisión de una demostración matemática, a los cuales se les agregó un corolario de política, de manera muy persuasiva (Dobb, 1975, pp. 80-81).

\section{Jean-Baptiste Say (1767-1832)}

Say establece una relación directa entre el valor y la utilidad al decir que "human industry, with the aid of capital and of natural agents and properties, creates every kind of utility, which is the primary source of value"6 $^{\prime 2}$ (Say, 1971, p. 284). Así, asume que la

6 "La industria humana, con la ayuda del capital y de los agentes y propiedades naturales,

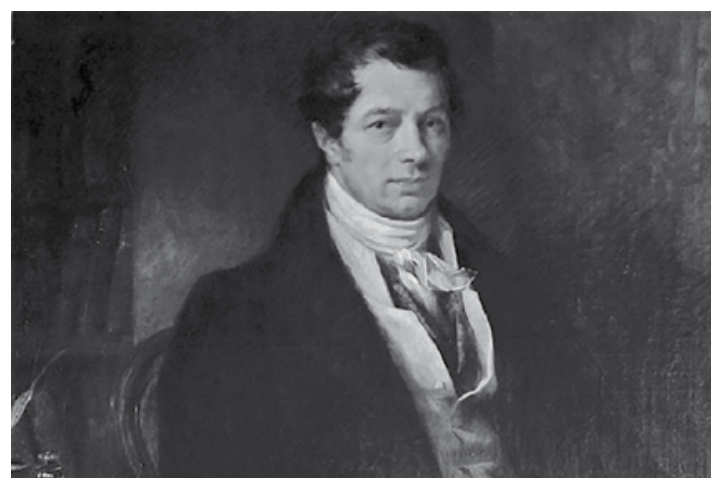

Jean-Baptiste Say

utilidad, entendida como la capacidad de uso, refleja el precio y sirve como fundamento de la demanda; en otras palabras, es la fuente de valor. Por otro lado, considera que el trabajo (la industria humana) es un insumo que prima por encima de otros, como el capital y la tierra, puesto que estos últimos son instrumentos para la producción de algún bien, mientras que el trabajo materializa la utilidad (el valor de uso).

En su libro A treatise on political economy, Say (1971: 31) no comparte la idea de que el único fundamento y medida correcta del valor de las cosas sea el trabajo, tal como lo piensa Smith. Además, piensa que Adam Smith cometió un error al pensar que "wealth is nothing more than labor accumulated; from which position he infers a second consequence equally erroneous that labor is the sole measure of wealth, or of value produced"7 (Say, 1971, p. 76); sin embargo, no menciona otros aspectos que podrían utilizarse a la hora de establecer el valor de un bien.

crea todo tipo de utilidad, que es la principal fuente de valor" (traducción hecha por los autores).

7 "La riqueza no es más que trabajo acumulado; desde qué posición infiere una segunda consecuencia igualmente errónea, a saber. que el trabajo es la única medida de riqueza o de valor producido" (traducción hecha por los autores) 


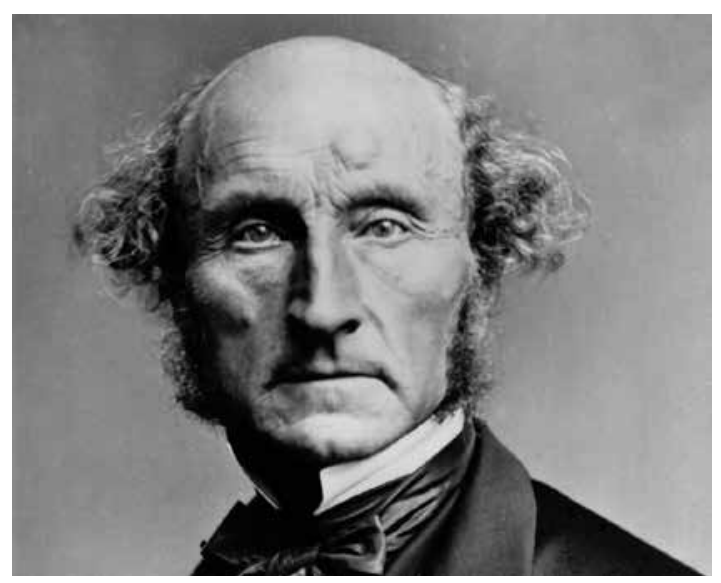

John Stuart Mill

\section{John Stuart Mill (1806-1873)}

John Stuart Mill fue un economista de origen inglés que trató la teoría del valor; sin embargo, la literatura le ha catalogado como una adición a la teoría de costos de Adam Smith o a la suma de componentes (Dobb, 1975, p. 138; Jaffe y Lusht, 2003, p. 31) e incluso como poco interesante (Dobb, 1975, p. 142). Empero, se observa una postura diferente en Caplan (1931) dentro de su libro Theories of value from David Hume to John Stuart Mill.

Mill logra establecer dos tipos de bienes con causas diferentes. El primero de ellos corresponde a aquellos que se pueden producir a voluntad, mientras que los segundos son aquellos que no comparten esta característica. Al respecto Mill anota:

demand and supply govern the value of all things which cannot be indefinitely increased; except that even for them, when produced by industry, there is a minimum value, determined by the cost of production. But in all things which admit of indefinite multiplication, demand and supply only determine the perturbations of value (como se citó en Caplan, 1931, p. 122) ${ }^{8}$.

8 "la demanda y la oferta gobiernan el valor de todas las cosas que no pueden aumentarse indefinidamente; excepto que incluso para
De esta manera, los costos de producción y la oferta y demanda son los determinantes del valor. Para los bienes producidos a voluntad, el valor de mercado oscila en torno a los valores naturales (Caplan, 1931, p. 118). Mill define el valor natural como aquel valor de escasez para ciertos periodos particulares del tiempo $y$, la generalidad de este, como el resultado de los valores de costo o de producción (Caplan, 1931, p. 118). Los costos producción están conformados por "as necessary, wages and profits, as occasional, taxes and any extra cost caused by a scarcity value of some of the requisites" (Caplan, 1931, p. 118). No obstante, el factor de trabajo juega el papel más importante, aunque no torna insignificante los demás valores (Caplan, 1921, p. 119). Adicionalmente, la creación de valor en Mill se establece la necesidad de que el objeto brinde alguna utilidad de uso y que para la consecución de dicho objeto se encuentre alguna dificultad (Caplan, 1931, p. 119).

Los aportes de Mill a la teoría del valor trabajo que, si bien comparte algunas características de Adam Smith, ofrece nuevos elementos de análisis al presentar diferentes explicaciones causales de valor y las condiciones para la creación de este.

\section{Karl Marx (1818-1883)}

Karl Marx (1818-1883) fue un economista de origen prusiano. Su importancia se debe a la creación conceptual del trabajo socialmente

ellas, cuando son producidas por la industria, existe un valor mínimo, determinado por el costo de producción. Pero en todo lo que admite una multiplicación indefinida, la demanda y la oferta solo determina perturbaciones del valor" (traducción hecha por los autores).

9 "según sea necesario, salarios y ganancias, como ocasionales, impuestos y cualquier costo adicional causado por el valor de escasez de algunos de los requisitos" (traducción hecha por los autores). 


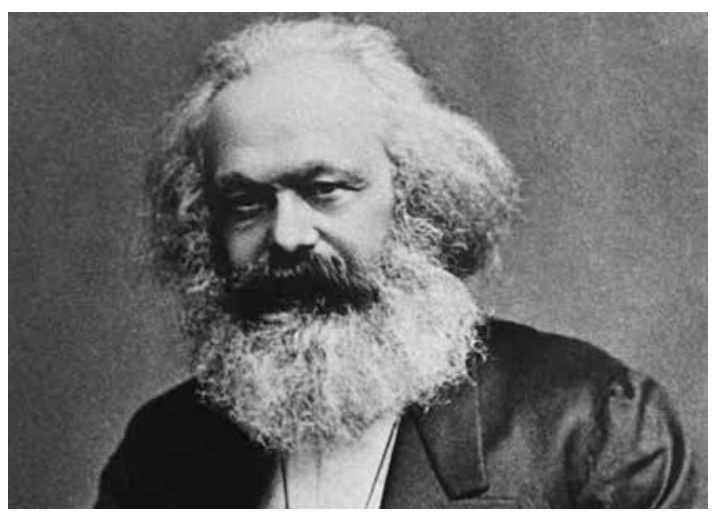

Karl Marx

necesario, el cual reformula la teoría del valor al establecer que el trabajo no crea el valor, sino que es el trabajo socialmente necesario y el intercambio los fundamentos de este. Su teoría se ubica en la explicación de la mercancía, la cual contiene tres dimensiones: el valor de uso, referido a la satisfacción de necesidades (Marx, 1981, p. 3); el valor de cambio, como la proporción de cambio entre mercancías (Marx, 1981, p. 4); y el concepto de valor que comprende la sustancia y magnitud de valor (Marx, 1981, p. 6).

El valor de cambio, al ser una expresión de la mercancía, es tan múltiple como mercancías existen (Marx, 1981, p. 6), "lo que caracteriza visiblemente la relación de cambio de mercancías es precisamente el hecho de hacer abstracción de sus valores de uso respectivos" (Marx, 1981, p. 5). Dicha abstracción recibe el nombre de trabajo abstracto; sin embargo, este no es suficiente explicación para la creación del valor. Para Marx, el valor estaba compuesto por la magnitud de valor y sustancia de valor; la primera es la medición de la segunda - comúnmente en tiempo- (Marx, 1981, p. 6). Con respeto a la sustancia de valor, para su medición se requiere que el trabajo humano debe estar igualado. Este problema no se puede resolver con el trabajo abstracto, ya que este es una generalización del trabajo concreto y no un procedimiento. Por esta razón, se crea el concepto de trabajo socialmente necesario, que se entiende como "aquel que se requiere para producir un valor de uso cualquiera, en las condiciones normales de producción y con el grado medio de destreza e intensidad de trabajo imperantes en la sociedad" (Marx, 1981, pp. 6-7). De esta manera, la sustancia de valor se torna como el trabajo promedio que la sociedad requiere para la creación de una mercancía, encontrándose atada a una realidad geotemporal específica.

Se debe agregar que, para la constitución de la mercancía es necesario que exista el intercambio. Por este medio se expresa el valor de una mercancía por la relación existente entre los valores de dos o más de ellas (Marx, 1981, p. 15). Al respecto, Marx anota:

la expresión de equivalencia de diversas mercancías las que pone de manifiesto el carácter específico del trabajo como fuente de valor, al reducir a su nota común, la de trabajo humano puro y simple, los diversos trabajos contenidos en las diversas mercancías (Marx, 1981, p. 18).

De este modo, al implementar una condición al trabajo humano se logra determinar qué tipo de trabajo crea valor. Por tanto, el trabajo socialmente necesario o el trabajo promedio de la sociedad es la única sustancia que puede crearlo. Finalmente, cabe destacar que para Marx el concepto de valor es diferente al de precio, ya que este último se define por las fuerzas de la oferta y de la demanda y no por el trabajo socialmente necesario (Cuevas, 2003, p. 37-38). En conclusión, Marx logra establecer varios conceptos y se posiciona como punto cúspide dentro de la teoría.

\section{Alfred Marshall (1842-1924): escuela neoclásica}

Para la segunda mitad del siglo XIX surgiría una escuela de pensamiento económico, a partir de la escuela marginalista, conocida como la escuela neoclásica. Su máximo exponente, Alfred Marshall, reformularía la idea 


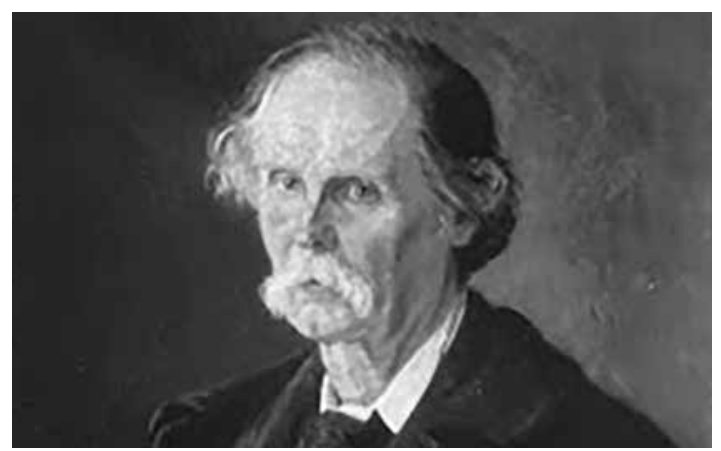

Alfred Marshall

sostenida por los clásicos que postulaba que el valor provenía del trabajo por parte de la producción, es decir, de la oferta; Marshall propone que el valor depende de las fuerzas de trabajo, oferta y demanda, costo marginal de producción y utilidad marginal, respectivamente. Esta teoría propuesta es conocida como la teoría dual del valor. Para realizar una analogía sobre la determinación del valor a partir de sus ideas, Marshall (1963) afirma en su más importante obra Principios de Economía que:

Discutir acerca de si el valor está determinado por la utilidad o por el coste de producción sería lo mismo que discutir acerca de si es la lámina superior de un par de tijeras o la inferior la que corta un trozo de papel. Es cierto que, cuando se mantiene una lámina fija y se corta moviendo la otra, puede decirse al pronto es la segunda lo que lo corta, pero la afirmación no es estrictamente exacta, y sólo puede disculparse si pretende ser meramente una explicación popular de lo que ocurre y no una afirmación estrictamente científica (Marshall, 1963, p. 289).

Marshall introdujo por primera vez el aspecto del tiempo en la teoría del valor y al análisis económico, en general, considerándose uno de sus más grandes aportes a la ciencia económica. Con esta introducción a la economía buscaba "trazar un hilo continuo que atraviese y conecte las aplicaciones de la teoría general del equilibrio de la demanda y la oferta de diferentes períodos de tiempo" (Marshall,
2005, p. 549). Esto permitió clasificar el valor en cuatro tipos, cada uno de ellos en una recta entre dos extremos de determinantes de costos: el primero, las fuerzas de oferta y demanda, con especial énfasis en esta última, en periodos cortos de tiempo y, segundo, la modificación de variables de producción en periodos largos de tiempo. De esta manera, el costo es determinado por una ponderación entre estos dos extremos en un momento de tiempo dado.

\section{John M. Keynes (1883-1946): escuela keynesiana}

John M. Keynes (1883-1946), precursor de la macroeconomía y de la economía heterodoxa, con lo que respecta con la teoría del valor, no hace énfasis ni un estudio especial en esta; sin embargo, en algunos fragmentos de su obra cumbre titulada Teoría General de la Ocupación, el Interés y el Dinero (1936) y a través de una lectura minuciosa, parece a simple vista evidenciar ser partidario del valortrabajo, al decir:

Es preferible considerar al trabajo, incluyendo, por supuesto, los servicios personales del empresario y sus colaboradores, como el único factor de la producción que opera dentro de un determinado ambiente de técnica, recursos naturales, equipo de producción y demanda efectiva. Esto explica, en parte, por qué hemos podido tomar la unidad de trabajo como la única unidad física que necesitamos en nuestro sistema económico, aparte de las de dinero y de tiempo (énfasis propio [Keynes, 2007, p. 191]).

No obstante, cabe aclarar que Keynes recalca cómo el trabajo no es el único factor de producción que interviene en el valor, sino que tiene que estar ayudado de otros elementos como la técnica, los recursos naturales o los resultados del trabajo pasado (Astarita, 2010, p. 11). Dicho lo anterior, aunque simpatiza "con la doctrina preclásica de que todo es 
producido por el trabajo" (Keynes, 2007, p. 190), solamente está reconociendo al trabajo como el único elemento que permite crear de manera física los bienes, mas no lo está estableciendo como el determinante de la fuente del valor (como lo constituye el precio), ni mucho menos de la ganancia capitalista.

En adición con lo anterior, Keynes introduce la escasez para explicar cómo esta incrementa el precio de los bienes por sobre "su valor", dando lugar así a la ganancia (Astarita, 2010, p. 6); esta afirmación, como lo plantea el economista Kicillof, significa que existe una "limitación en la producción de bienes de capital con respecto a las necesidades existentes de dichos bienes, y que esa limitación se manifiesta en un incremento del precio de sus 'servicios'" (Kicillof, 2007, p. 440). Concluye, así, que la escasez (o lo contrario, la abundancia) son factores que permiten determinar los precios de los bienes, junto con el costo en trabajo que se generó para su elaboración.

En conclusión, Keynes deja en evidencia su interés en esta última cuestión de cómo la escasez del capital permite determinar la ganancia, siendo un postulado contradictorio con la misma teoría del valor trabajo, ya que el trabajo debería ser tanto la fuente del valor como de la ganancia.

\section{Piero Sraffa (1898-1983): escuela neo-ricardiana}

Piero Sraffa (1898-1983) tuvo a su cargo la edición de obras de Ricardo y su paso como profesor en Cambridge lo llevó a unirse al grupo de los neoricardianos que rechazaban la teoría marginalista. Su aporte a la teoría del valor se encuentra en su libro Production of Commodities by Means of Commodities, donde se enfoca en mostrar por medio de ecuaciones simultáneas los precios relativos. La unidad de medida aquí pasa a ser la "mercancía" o producto y no el trabajo, es decir, parte del valor de cambio de la mercancía (Potón, 2004, p.

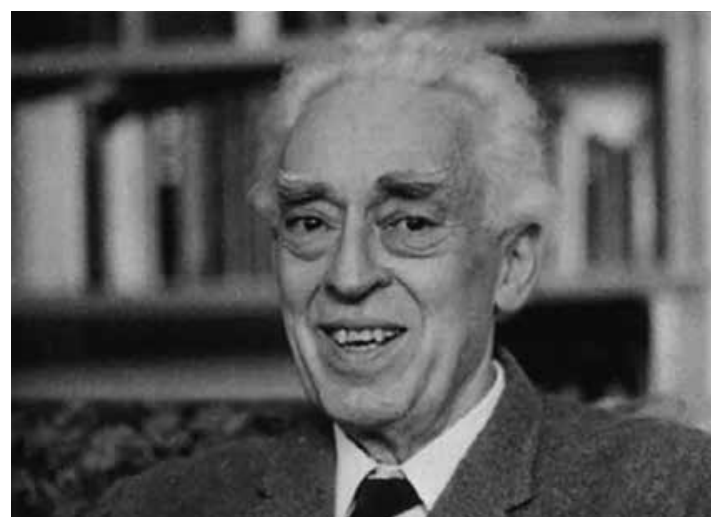

Piero Sraffa

79). Esto lo explica a través del trigo y el hierro, nombrando a este modelo como "métodos de producción” (Sraffa, 1975, p. 79-80). Estos productos los denomina como productos básicos, que son los únicos que pueden cambiar los precios y la tasa de beneficio (Dobb, 1975, p. 282). Concluye que a partir del valor de cambio se puede dar nuevamente esta transformación gracias al método de producción (Sraffa, 1975, p. 80-81).

Sraffa introduce, además, los términos de "mercancía compuesta" y "mercancía patrón". El primero se refiere al conjunto de precios y los salarios y el segundo se refiere a la mercancía industrial, que en su conjunto compone el sistema patrón (Sraffa, 1975, p. 80). Con esto, concluye que la tasa de beneficio está determinada por la cantidad de mercancías y, a la par, si los salarios se reducen constantemente, la tasa de beneficios se asocia directamente con su decrecimiento (Potón, 2004, p. 81).

Para finalizar, Sraffa realiza su teoría por medio de suposiciones, aunque resalta que su sistema funcionará en el sistema económico real (Potón, 2004, p. 81). Su teoría es una crítica a la teoría marginalista, que se resalta en el prefacio de su libro y a su pertenencia en el grupo neoricardiano. Además, resolvió el problema central de la economía clásica de Ricardo con su sistema de ecuaciones, dando a resolver la fluctuación de los precios si surgían cambios en la mercancía (Dobb, 1975, p. 288). 


\section{Escuela postkeynesiana}

La mayoría de los autores postkeynesianos no centraron sus obras en el estudio de la teoría del valor-trabajo, por lo que no se establece un concepto uniforme dentro de la escuela; sin embargo, es posible rastrear algunos elementos conceptuales en varios autores.

La primera de ellas es Joan Robinson (1903-1983), quien acude a la microeconomía para establecer una relación entre la teoría de la productividad marginal y el trabajo. Es claro que para ella, el salario es la representación monetaria del trabajo, además plantea que el valor monetario de la fuerza de trabajo de una persona está definido por su productividad marginal y contribución a la sociedad; sin embargo, en el siguiente ejemplo deja en entredicho la objetividad del cumplimiento de la teoría del valor-trabajo de la fuerza laboral y cuestiona la existencia de una teoría de la distribución en la asignación de los salarios de los trabajadores en relación a su contribución social.

El sueldo de un profesor de economía mide su contribución a la sociedad y el sueldo de un basurero también mide la contribución que éste hace. Desde luego, la doctrina resulta muy reconfortante para los profesores de economía, pero tengo la sospecha de que se trata de otra tautología (Robinson, 2015, p. 212).

Para Maurice Dobb (1938, p. 15), la esencia de la producción está dada por el trabajo que hay detrás de ella y comparte la idea de que las personas asignan el valor a las cosas basándose en el trabajo que involucra su creación. No obstante, no apoya la noción de Ricardo que menciona que el intercambio de las mercancías es posible gracias al valor que conserva cada una, valor que es asignado por el trabajo en que se incurrió durante su creación, puesto que para él no hay homogeneidad en él. Por otro lado, cree que lo correcto es que la mezcla entre el capital y el trabajo defina el valor de las cosas: "los valores del mercado son iguales a los salarios más el tanto normal de ganancias sobre el capital empleado" (Dobb, 1938, p. 18). Así, el salario juega el papel de representación monetaria del trabajo y las ganancias reflejan la proporción de capital.

Michał Kalecki (1899-1970) es considerado como un economista marxista y posterior a Keynes, quien, en cuanto a su teoría del valor, se aleja de la concepción del valor-trabajo y se encamina con exclusividad a la determinación de los precios. La visión de Kalecki se caracteriza por considerar que la economía moderna se desarrolla en un contexto de competencia imperfecta, en donde se concentra el capital y el dominio de los mercados (Gallo, 2011, p. 16; Sawyer, 1985, p. 20). De esta manera, "the oligopolistic nature of industries led Kalecki to the view that the mark-up of price over costs was determined by the degree of monopoly"10 (Sawyer, 1985, p. 20).

Igualmente, Kalecki determinó dos tipos de precios con base de dos clases de productos; en los productos elaborados o semielaborados su precio es, en gran medida, determinado por los costos de producción, en cambio, en las materias primas su precio se determina por la demanda (Sawyer, 1985, p. 20; Gallo, 2011, p. 20-21). Esta distinción es causada gracias a que "the supply of finished goods is seen as being elastic (...) as a result of existing reserves of productive capacity, and with costs roughly constant with respect to output changes"11

10 "La naturaleza oligopolística de las industrias llevó a Kalecki a la opinión de que el aumento del precio sobre los costos estaba determinado por el grado de monopolio" (traducción hecha por los autores).

11 "La oferta de bienes terminados se consideraba elástica (...) como resultado de las reservas de capacidad productiva, y con costos aproximadamente constantes con respecto a los cambios de producción" (traducción hecha por los autores). 
(Sawyer, 1985, p. 21), mientras que "la oferta de las primeras [materias primas] se considera inelástica en el corto plazo" (Gallo, 2011, p. 20). En cuanto a los productos elaborados y semielaborados, su costo de producción es la suma de los costos de insumos, salarios y de monto de ganancias (Gallo, 2011, p. 16) y, respecto a este último, se define por el grado de monopolio, conocido también con el nombre de mark up, el cual define la fijación de los precios (Gallo, 2011, p. 16-17; Sawyer, 1985, p. 21). De esta manera, los precios son generalmente superiores a los costos primos y dicha diferencia genera el beneficio económico.

Sawyer (1985) atribuye otros dos factores presentes en Kalecki para la determinación de los precios en productos elaborados o semielaborados: "The two factors which are the major influences on the firm's price decision are its average prime costs and the prices of other firms producing similar products"12 (Sawyer, 1985, p. 21), es decir, el precio de estos productos se encuentra determinado más allá de sus costos de producción, puesto que puede influir la demanda y los precios de la competencia (en el caso de que existiesen). Por lo tanto, la teoría de precios de Kalecki se diferencia de la teoría valor trabajo, aunque, si bien, el costo de producción -entre ellos los salarios y el trabajo- juega un papel importante en la determinación del precio, Kalecki incluye nuevos factores de análisis.

\section{Conclusiones}

La teoría del valor-trabajo, en cuanto a enfoque de pensamiento económico, tiene un amplio desarrollo teórico, más allá del que se pudo abarcar en estas páginas. Sin embargo, este trabajo pretendió reunir algunas de las concepciones económicas más importantes dentro de esta vertiente.

Es interesante, como primera instancia, destacar el papel de Karl Marx para la teoría de valor y como este economista representa un punto de inflexión en ella, puesto que logra introducir grandes modificaciones hasta su momento $\mathrm{y}$, además, representa igualmente uno de los últimos grandes economistas que priorizaron el estudio de la teoría del valor trabajo, ya que después de Marx llegaron otros economistas, para los cuales, ni el trabajo dentro de la teoría del valor ni esta misma ocuparon un interés y en cambio se pondera

* "Los dos factores que son las principales influencias en la decisión de precio de la empresa son sus costos primos promedio y los precios de otras empresas que producen productos similares" (traducción hecha por los autores). con otras variables. A partir de este punto, la teoría del valor-trabajo empieza a perder su importancia en el mundo académico.

No obstante, es necesario recalcar una característica que, generalmente, estuvo común en la mayoría de las concepciones de los autores; la presencia de la oferta y la demanda, la cual, por su propia capacidad, podría llegar a generar un nuevo valor de mercado diferente al creado por el trabajo, llámese este valor de mercado en Petty, valor de cambio de Ricardo - determinado por la escasez-, perturbaciones del valor natural en Mill, precio en Marx y Keynes y teoría dual en Marshall. De este modo, es posible determinar que la teoría del valor-trabajo no es excluyente con las fuerzas del mercado, sino que más bien son conceptos complementarios. En tal sentido y contrariamente a lo que se pueda pensar, la teoría del valor-trabajo no fue una categoría hermética con una única variable: el trabajo.

En segunda instancia, se presentan algunas diferencias con respecto a la unidad de medida de valor, puesto que se pueden evidenciar dos extremos teóricos: 1) la única medida 
de valor es el trabajo, posición mantenida por Marx con el concepto de trabajo socialmente necesario y 2) la unidad de medida es el resultado de ponderar varias variables, la cual es una posición que comparten la mayoría de los economistas estudiados; siendo esta defendida desde posiciones menos radicales como la Smith o Ricardo, hasta posiciones completamente radicales como la de Marshall, Kalecki o incluso Keynes, en quienes el trabajo puede llegar a tener un papel casi minoritario o secundario. Adicionalmente, este fenómeno no es exclusivo de una temporalidad determinada, sino que ha estado presente desde el inicio conceptual de la teoría valor trabajo, ya que, por ejemplo, para Petty se determina en conjunto entre trabajo y otro elemento como la tierra y para Smith por los salarios, el beneficio y la renta, pero, en contraste con lo anteriormente dicho, sí se puede establecer que la reducción de la importancia del trabajo para la ponderación es propia de economistas posteriores al Marx.

Igualmente, cabe volver a mencionar como, dentro de las concepciones teóricas examinadas, la postura de Marx es la más próxima a ser una teoría autopoética en el sentido de que su concepción toma como única medida al trabajo, pero lo condiciona y limita al identificarlo exclusivamente como el trabajo socialmente necesario. De esta manera, la creación del valor se encuentra atada a realidad geotemporal específica, de tal forma que se pueden efectuar varias comparaciones con diferentes tipos de trabajo. Este aspecto comúnmente no fue especificado por los demás autores y aún más en los autores posteriores a Marx, los cuales, solamente resumen al trabajo bajo las categorías de salario, en donde no se especifican más detalles.
En última instancia, se ha mostrado que la teoría del valor trabajo tuvo un amplio desarrollo conceptual a medida que pasaba entre los autores, así como algunas de las cualidades del valor estuvieron presentes desde las primeras concepciones, mientras que otras se fueron dando en el desarrollo y expansión conceptual. El desarrollo de la teoría valortrabajo, en síntesis, se puede establecer como un desarrollo más o menos homogéneo, con una notable diferenciación en Marx en donde establece una posición más radical en cuanto a la unidad de medida de valor. Igualmente, el estudio histórico de la teoría del valor nos permite observar el progreso teórico-histórico que ha tenido esta temática y nos permite cuestionar el por qué en la comunidad académica se nos muestra como un producto acabado y sin profundidad.

El estudio de la teoría del valor-trabajo puede llegar a ser de utilidad en casos de la vida práctica o en el campo del estudio académico, siendo en este último donde se presentan las mayores complejidades teorías para repensar una teoría en el siglo XXI, en donde las circunstancias del statuo quo son diferentes a las pensadas por los economistas estudiados. Este es un reto que se tendrá que asumir en próximas investigaciones de la teoría del valor-trabajo.

A modo de síntesis, a continuación, se presentan dos tablas con los autores que se estudiaron en este trabajo de investigación, recopilando las principales concepciones $y$ aportes a la teoría, de tal forma que se pueda identificar cómo fue la continuidad, qué elementos se agregaron o quedaron estáticos y hasta qué momento histórico se destaca su importancia. 


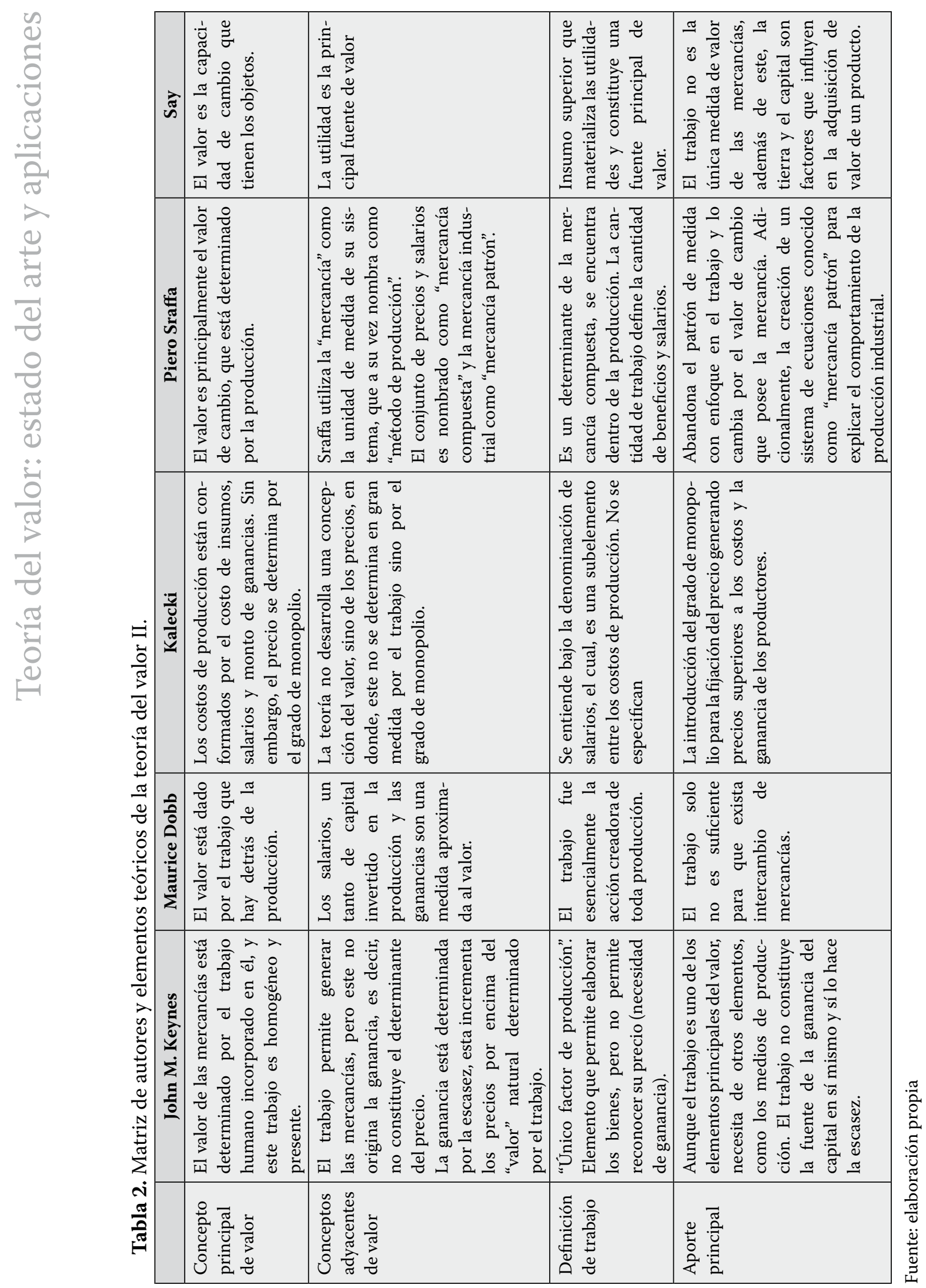




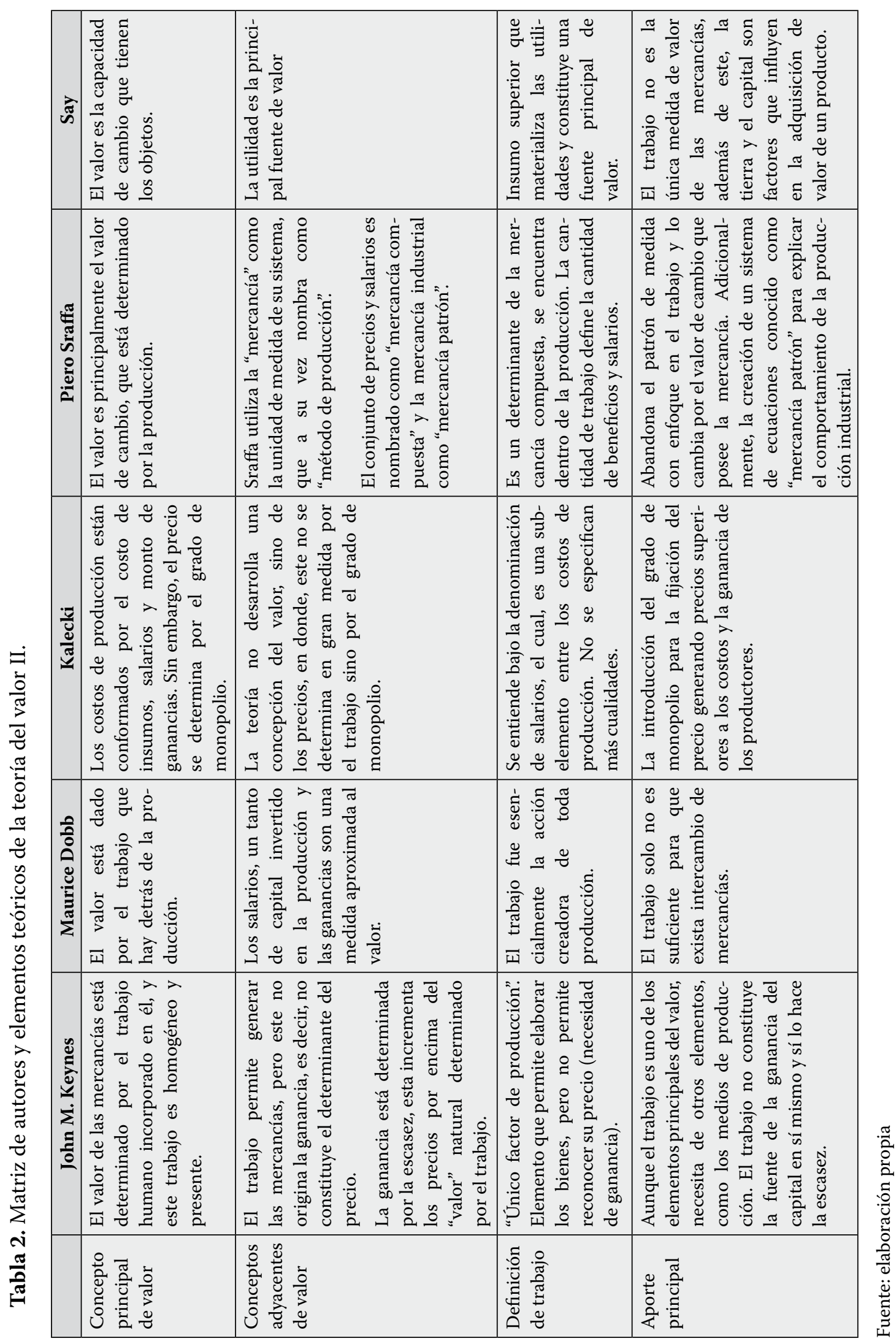




\section{Referencias}

Astarita, R (enero 5, 2010) ¿Keynes partidario del valor trabajo? [Entrada de blog]. Recuperado de: https://rolandoastarita. blog/2010/10/28/\%C2\%BFkeynes-partidario-del-valor-trabajo/

Caplan, B. (1931). Theories of value from David Hume to John Stuart Mill. Recuperado de: http://digitool.library.mcgill. $\mathrm{ca} / \mathrm{R} /$-?func $=$ dbin-jump- full\&object_ id $=136510 \&$ silo_library $=$ GEN01

Cuevas, H. (2003). Valor y sistemas de precios. Bogotá, Colombia: Universidad Nacional de Colombia

Díaz, Y. (2014). William Petty: un clásico de la Economía Política. Su aportación a las categorías económicas. Revista Debate Económico, volumen (3). 126-127.

Dobb, M. (1938). Introducción a la economía. (A. Castro, Trad.). México DF, México: Fondo de Cultura Económica.

Dobb, M. (1975). Teoría del valor y de la distribución desde Adam Smith: Ideología y teoría económica. (R. Cendrero trad). Argentina: Siglo XXI Argentina Editores S.A.

Dooley, P. (2005) The Labour Theory of Value. Londres, Inglaterra: Routledge.

Gallo, M. (2011). Marx, Keynes y Kalecki: sus conexiones en la teoría la distribución del ingreso, el valor de cambio y la naturaleza del capital. En, pensar un mejor trabajo, acuerdos, controversias y propuestas. Simposio llevado a cabo en el X Congreso Nacional de Estudios del Trabajo por la asociación argentina de especialista en estudios del trabajo. Recuperado de: http:// nulan.mdp.edu.ar/1382/1/01221.pdf

Guillén, A. (1976). Adam Smith y la teoría del valor trabajo. Revista Latinoamericana de economía, 6(25), 18-21.

Jaffe, A. y Lusht, K. (2003). The History of Value Theory: The Early Years. En Sirmans, C, et al. (eds). Essays in Honor of William N.
Kinnard, Jr. (pp. 3-44). New York, EEUU: Springer Science+Business Media.

Kaulla, R. (1940). Theory of the Just Price: A Historical and Critical Study of the Problem of Economic Value (R. D. Hogg, trad.). Londres, Gran Bretaña: Unwin Brothers Limited Working. Recuperado de https://dspace.gipe.ac.in/xmlui/handle/ 10973/32904

Keynes, J. (2007). Teoría General de la Ocupación, el Interés y el Dinero (E. Hornedo, trad.). México: Fondo de Cultura Económica.

Kicillof, A. (2007). Fundamentos de la Teoría General: Las consecuencias teóricas de Lord Keynes. Buenos Aires, Argentina: Eudeba.

Marshall, A. (1963). Principios de Economía: un tratado de Introducción. (E. de Figueroa, Trad.) Madrid, España: Editorial Aguilar S.A.

Marx, K. (1981). El capital: Crítica de la economía política (W. Roces, trad.) (14 edición). Bogotá, Colombia: Fondo de Cultura Económica

Moreno, Á., Rendón, J. (2005). La corriente objetiva y la corriente subjetiva: Un debate entre economía política y simplemente economía: una perspectiva crítica. Entramado, 1(2), 48-66. Recuperado de https://www.redalyc.org/comocitar. oa?id=265420471006

Potón, R. (2004). La teoría del valor y el pensamiento de Sraffa. Invenio, 7(12). P. 77-84.

Robinson, J. (2015). La segunda crisis de la teoría económica. Revista de Economía Crítica (19) 204-213. Recuperado de http://www.revistaeconomiacritica.org/ sites/default/files/revistas/Revista_Economia_Criti ca_19.pdf

Roll, E. (1973). Historia de las doctrinas económicas. México: Fondo de Cultura Económica. 
Sawyer, M. (1985). The Economics of Michat Kalecki. New york, Estados Unidos: M. E. Sharpe, Inc. Recuperado de www.jstor. org/stable/4379705

Say, J. B. (1971). A treatise on Political Economy (Quinta ed.). New York, Estados Unidos: Augustus M. Kelley, Publishing

Sewall, H. (1901). The Theory of Value before Adam Smith. Publications of the American Economic Association, 2(3), 3rd series, 1-128. Recuperado de www.jstor.org/ stable/2485740
Sir William Petty (1963). The Economic Writings of Sir William Petty (C. Hull, ed). New York, Estados Unidos: Kelley.

Smith, A. (1994). la riqueza de las naciones. Madrid, España: Alianza editorial S.A

Sraffa, P. (1975). Producción de mercancías por medio de mercancías: preludio a una crítica de la teoría económica (L. A. Rojo, trad.). Barcelona, España: Oikos-tau. 